\title{
Spongy glio-neuronal dystrophy: a degenerative disease of the nervous system
}

\author{
IAN J. HOPKINS AND BRIAN TURNER
}

\author{
From the Department of Paediatrics, University of Melbourne, and \\ Royal Children's Hospital, Melbourne, Victoria, and The Children's Medical Research \\ Foundation, Royal Alexandra Hospital for Children, Camperdown, N.S.W., Australia
}

SUMMARY A case of spongy glio-neuronal dystrophy is reported. The clinical features included mental and motor retardation in early infancy after a normal birth, and from the age of 4 years increasingly severe attacks of multifocal epilepsy, with prolonged postictal coma, myoclonic twitching and nystagmus, and at times hemiplegia for one to two weeks. Acidosis was present during the periods of postictal coma. Jaundice occurred several days before death at the age of 5 years. Neuropathological examination revealed severe spongy degeneration in the thalamus and dentate nucleus, and the cerebral cortex to a lesser degree, with sparing of the white matter. There was partial necrosis of liver cells and in some areas regenerative nodules. Possible aetiological factors linking the cerebral and hepatic pathology are discussed.

Of the progressive degenerative diseases affecting the central nervous system in childhood the neuronal storage disorders and leukodystrophies are relatively well defined both with respect to clinical features and pathological changes. In contrast, there is a poorly classified and heterogeneous group of conditions among the degenerative diseases of the nervous system in childhood, and Alpers' disease (Alpers, 1931) or 'progressive infantile poliodystrophy' (Christensen and Krabbe, 1949) falls into this category. Klein and Dichgans (1969) reported the clinical and pathological features of three siblings with a condition closely related to Alpers' disease and which they called 'familial juvenile glioneuronal dystrophy'. More recently, Jellinger and Seitelberger (1970) have reported similar cases in earlier childhood, using the descriptive term 'spongy glio-neuronal dystrophy in infancy and childhood'. They reviewed previously reported cases of progressive poliodystrophy and suggested that approximately two-thirds of the cases are symptomatic of heterogeneous causal factors, mainly oxygen deprivation or circulatory disorder. Only in 24 cases did clinicopathological data fail to disclose evidence of exogenous or other known noxious factors. They considered this idiopathic type of progressive grey matter degeneration to be a distinct entity with the pre sumed basic pathology affecting the glioneuronat unit. In view of the prominent histological change of vacuolation they suggested the name "spongye glio-neuronal dystrophy' be used for these cases.

In this paper we report the clinical and pathological features of a further case of spongy glioneuronal dystrophy and discuss possible aetiological factors.

\section{CASE HISTORY}

J.M. (R.C.H. U.R. 203987) presented with nonspecific findings of mental and motor retardation in early infancy, and from the age of 4 years had increasingly severe attacks of multifocal epilepsy, with prolonged postictal coma, myoclonic twitching, and nystagmus. She died at the age of 5 years. Jaundice was present shortly before her death.

She was the second child of unrelated Caucasian parents neither of whom had a family history of neurological or mental illness. Her only sibling, a brother, was born by low forceps delivery after a normal pregnancy and labour. His birth weight was $3.7 \mathrm{~kg}(8 \mathrm{lb} .2 \mathrm{oz}$.) and, apart from being slightly irritable for the first 24 hours, was thought to be normal in early infancy. However, after several months it was apparent that his developmental pro- 
gress was slow. He learned to roll from side to side but never sat alone or crawled. During the second year of life he became very suntanned, and this persisted through an English winter. Pigmentation was most marked on exposed and pressure areas. He had recurrent severe upper respiratory tract infections and when nearly 3 years of age died suddenly during such an episode. Post mortem examination showed bronchopneumonia but neuropathological examination was not carried out.

The pregnancy with J.M. was also uneventful except for terminal hypertension which led to induction of labour. The delivery and neonatal periods were uneventful. Her birth weight was $3,240 \mathrm{~g}$. After several months it was apparent that her developmental progress was also markedly delayed. She did not sit alone until approximately 2 years of age and at no stage walked alone or had recognizable speech. However she would move about the floor by rolling. She showed some interest in toys.

When first examined by one of the authors (I.J.H.) at $\mathbf{1 4}$ months of age there was generalized hypotonia with brisk tendon reflexes. Tremulous movements of the hands were noted on intentional movements and when she became excited or agitated. Examination of the cranial nerves revealed no abnormality. The head circumference was $44.5 \mathrm{~cm}$, and it remained on the 3 rd centile thereafter.

There was very little progress in motor and speech development over the ensuing two years. The major problem in her management was recurrent respiratory infections with mild dehydration. Also, certain foods (roast meat and vegetables, fried chipped potatoes, chocolate cake) appeared to cause anorexia and loose reddish brown stools. At the age of 4 years 3 months her first episode of status epilepticus occurred, with focal left-sided convulsions. The postictal period was characterized by prolonged coma, nystagmus, myoclonic twitching - of the face and hands particularly-and acidosis. The blood $\mathrm{pH}$ was $7 \cdot 23, \mathrm{PCO}_{2} 23 \mathrm{mmHg}$, base excess $-16 \mathrm{~m}$-equiv/ 1., standard bicarbonate $13 \mathrm{~m}$-equiv/l., total $\mathrm{CO}_{2}$ $10.3 \mathrm{mM} / 1$. Serum lactate and pyruvate were normal. Her condition slowly improved after a week, and two weeks later she had recovered to her previous state. Up to the time of her terminal admission six months later she had several episodes of a similar nature commencing with focal convulsions, sometimes left sided and on other occasions right sided, followed by a variable period of coma of up to two weeks' duration. During this time diffuse myoclonic twitching of facial, trunk, and limb muscles, and coarse irregular nystagmus were present. In these later attacks the degree of acidosis was only mild, although this was treated energetically with appropriate intravenous therapy. Before attacks her parents thought she passed more urine than was usual, but she did not drink excessively. Her urine had a rather pungent odour at these times. After some attacks she was hemiplegic for a period of a week or two, corresponding with the lateralization of focal convulsions. She was not acidotic apart from the postictal periods. During her terminal admission lasting several months many such episodes of status epilepticus occurred. At this time it was apparent that her vision, which had previously seemed relatively normal, was markedly impaired but she still recognized her parents' voices. This impairment of vision appeared to be of cortical type, as pupillary reflexes and funduscopic examination were normal. Jaundice was first noted several days before her death at the age of 5 years and 1 month.

INVESTIGATIONS The following tests were carried out, many of them on several occasions, and were normal: full blood examination, serum sodium, potassium, chloride, urea, calcium, magnesium, blood sugar, lactate and pyruvate, uric acid, toxoplasma antibody titre, cytomegalovirus complement fixation antibody titre, immunoelectrophoresis, urine for metachromatic material, coproporphyrin, porphobilinogen and aminolaevulinic acid, radiographs of skull and long bones.

Serum ammonia was 89,92 , and $94 \mu \mathrm{g} \%$ on three separate occasions during the second and third years of life. Urine chromatography was normal on two occasions but on other occasions showed a slight increase in one or other of lysine, glycine, cystine, glutamine, valine or methionine, and $\beta$ aminoisobutyric acid. Serum amino acid analysis showed no consistent abnormality. Pneumoencephalography showed slight generalized ventricular dilatation. Electroencephalography showed mild increase in slow components in early infancy but in the last year of life several recordings contained persistent high voltage slow delta activity of 1 to $3 \mathrm{~Hz}$ with some superimposed fast components, sharp waves, and spikes. Variable lateralization of the high amplitude slow delta activity occurred in different episodes of epilepsy. Examination of the cerebrospinal fluid (CSF) several months before death showed a mild elevation of protein $(53 \mathrm{mg} / 100 \mathrm{ml}$.), but the colloidal gold curve was normal. Earlier CSF examination (cells, protein, sugar, colloidal gold, and WR) at 12 months of age had been normal. One day before her death serum bilirubin was $16.4 \mathrm{mg} /$ $100 \mathrm{ml}$., SGOT $156 \mathrm{SF}$ units, total serum proteins $4.8 \mathrm{~g} / 100 \mathrm{ml}$., and alkaline phosphatase $30 \mathrm{KA}$ units.

PATHOLOGY Necropsy was carried out five hours after death. The only macroscopic abnormalities noted were in the brain and liver. The brain weighed 


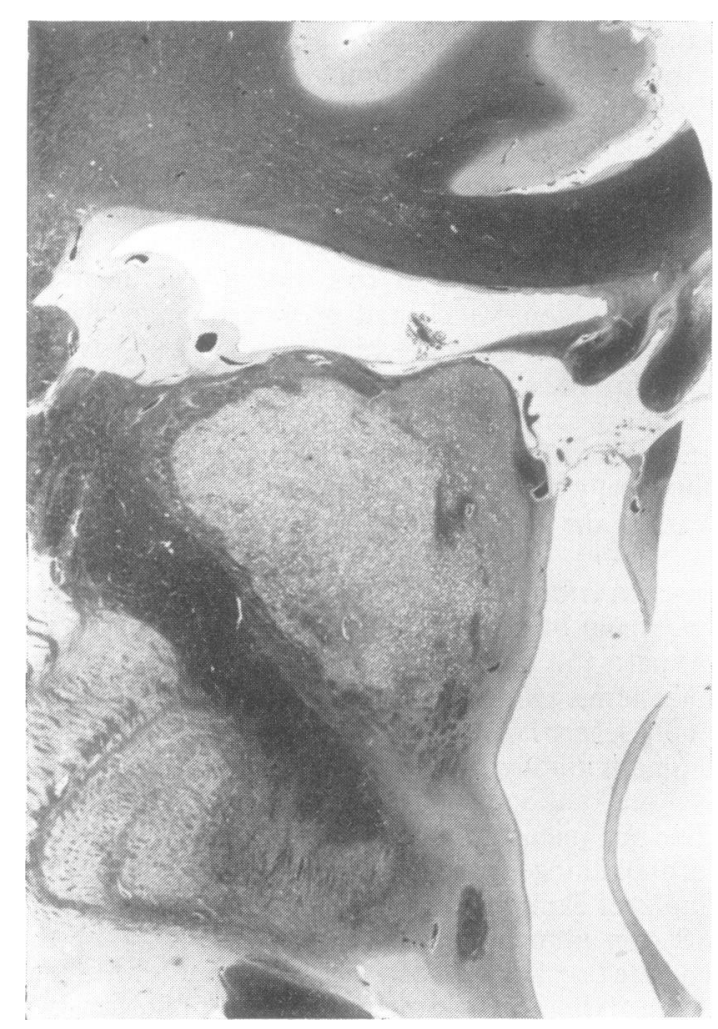

FIG. 1. Low power view of thalamus and adjacent structures showing spongy degeneration present in the thalamus.

$1,090 \mathrm{~g}$. The left frontal lobe was removed for chemical study. After fixation in formol saline the right hemisphere weighed $388 \mathrm{~g}$ and the brain-stem and cerebellum $105 \mathrm{~g}$-weights which are considerably reduced for a child of 5 years. The meninges on the lateral surface of the hemispheres and at the base of the brain were somewhat thickened and opaque. The gyral pattern of the hemispheres appeared normal but there was generalized narrowing of the convolutions which was most marked in the occipital regions. On section, the cerebral cortex was somewhat pale and in some areas thinner than normal. The atrophic foci were more frequent in the occipital region. The central white matter looked normal but felt abnormally firm, particularly in the occipital region. The corpus callosum was normal. The basal ganglia and ventricular system appeared normal but some atrophy of the thalamus was present and appeared more marked on the left. The brain-stem was somewhat smaller than normal. No pigment was seen in the substantia nigra; the pontine nuclei were smaller than normal and the pyramidal tracts were poorly myelinated in the medulla. The right cerebellar hemisphere was smaller than the left and the vermis was shrunken.

Histological examination was obtained of representative blocks of brain embedded in celloidin/ paraffin and stained by routine methods. The meninges showed some diffuse fibrous thickening but the pial vessels were unremarkable.

Diffuse degenerative changes were present throughout the cerebral cortex with focal accentuations. The most marked changes were present in the posterior frontal regions where there was a spongy change in the superficial and middle cortical layers with neuronal loss and gliosis but little blood vessel pro- $\vec{\circ}$ liferation. In the less severely affected regions the spongy change was most marked in the superficial layers. The nerve cells were reduced in number in all

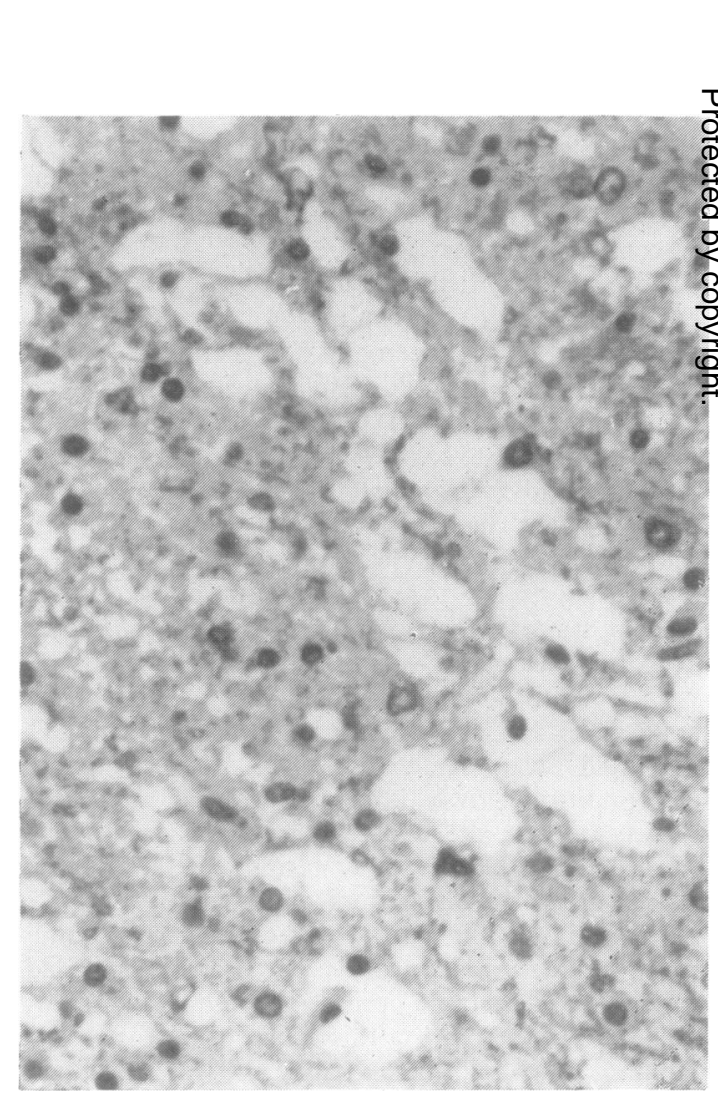

FIG. 2. Thalamus showing spongy change consisting of clear spaces of varying size with sharply defined borders. $\times 320$. 


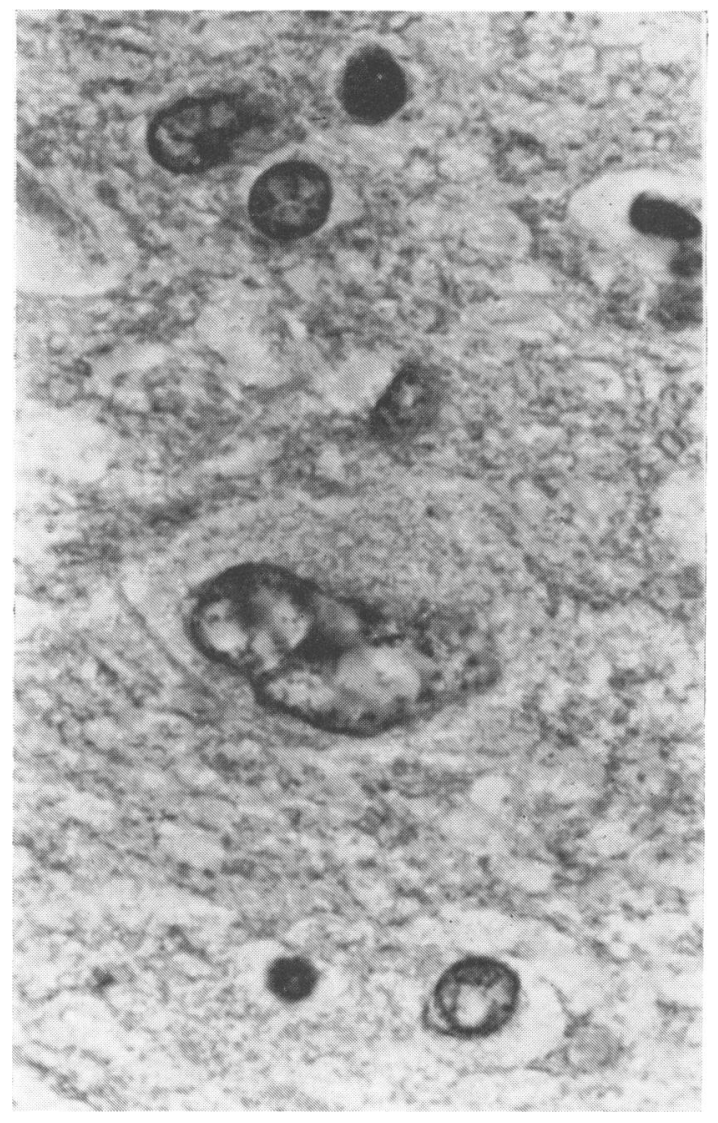

FIG. 3. Occasional giant bizarre astrocyte nuclei seen in areas of spongy change. $\times 800$.

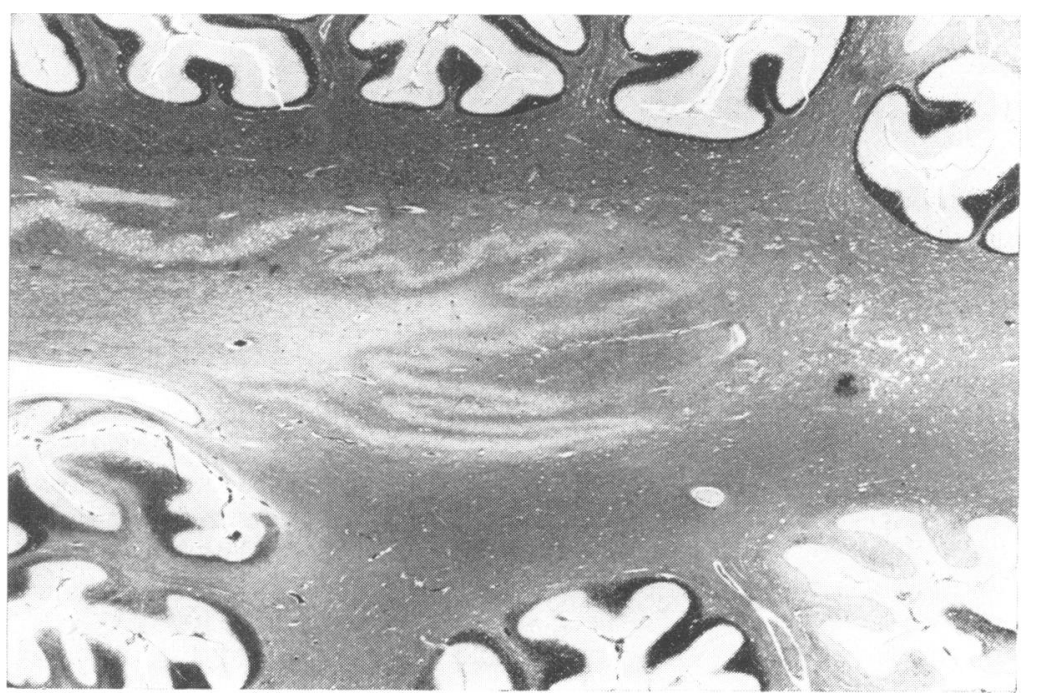

areas; the remaining nerve cells showed shrinkage and hyperchromasia in some regions, while in others they were normal. Neuronophagia was not observed.

Astrocytic proliferation accompanied the cortical degeneration. The astrocyte nuclei were $9-15 \mu$ in diameter and were generally rounded with clear nucleoli. In some regions protoplasmic astrocytes were present. No glycogen granules were demonstrated within the astrocytes. Rod type microglia were present in all cortical areas examined. Frozen sections stained with Scharlach $\mathbf{R}$ revealed small amounts of neutral fat accumulated within the perivascular spaces.

There was neuronal loss and gliosis in the Sommer sector of the hippocampus. The myelin in the central white matter stained normally except for some mild pallor in relation to the most severely affected areas of the cortex. In these areas some protoplasmic astrocytes had formed, while in the remainder of the white matter a general increase in the numbers of the astrocyte nuclei was found.

Marked spongy degeneration was present in the thalamus and the subthalamic nucleus (Fig. 1). The hypothalamus, zona incerta, substantia nigra, globus pallidus, caudate, and putamen were spared. The spongy change consisted of a series of clear spaces of varying size with sharply defined borders (Fig. 2). A nucleus which appeared to be of astrocytic type often projected into the space and occasionally fine filaments spread into or across the space from the region of this nucleus. The interstitial tissue showed degenerating nerve cells and myelin with increased numbers of astrocyte nuclei. Occasional giant bizarre astrocyte nuclei were present in these areas (Fig. 3).

FIG. 4. Low power view of the dentate nucleus. Methasol fast blue. Atrophy of the dentate nucleus with vacuolar change in the superior folium. Myelin loss is present in the hilum of the nucleus. 


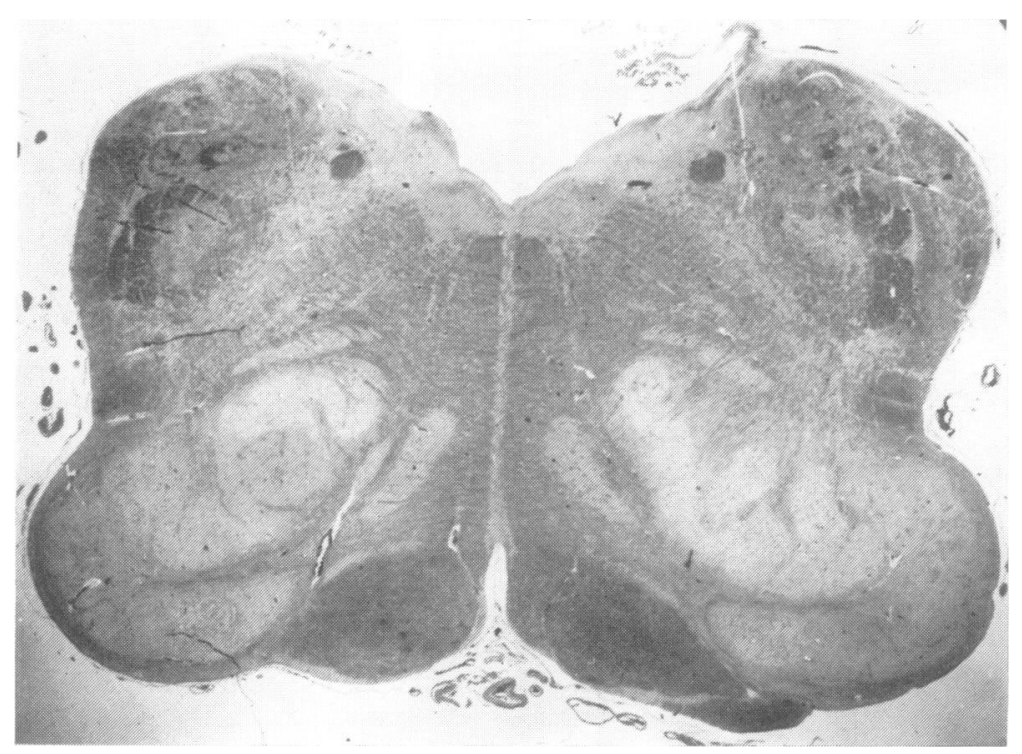

FIG. 5. Low power view of the medulla oblongata. Methasol fast blue. Marked swelling of the inferior olivary nuclei with some pallor of the myelin in the hilum of the nuclei.

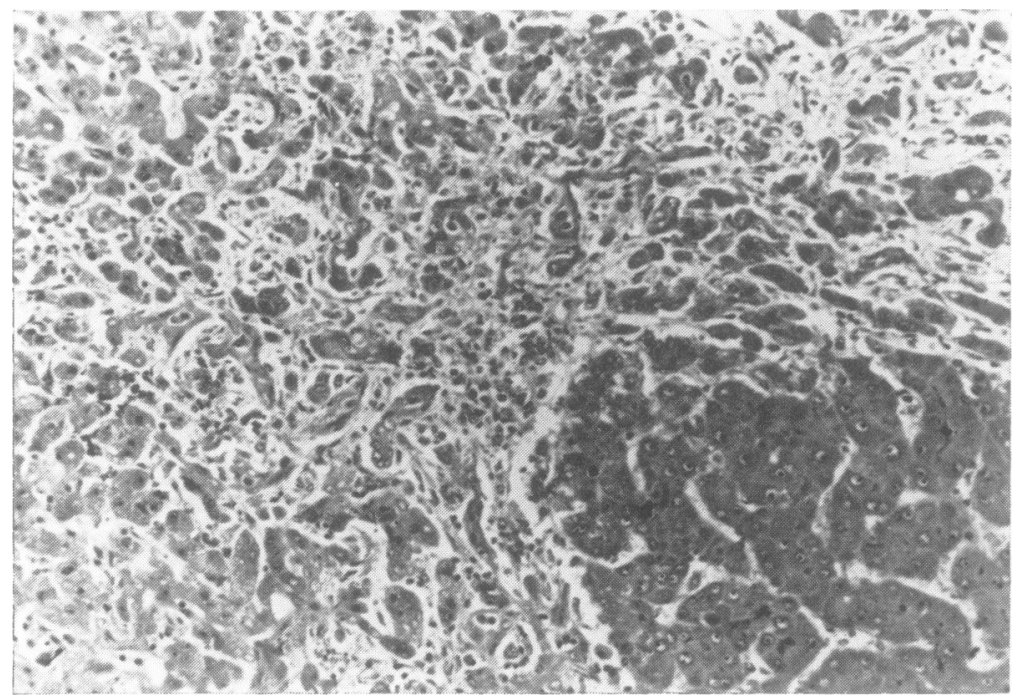

FIG. 6. A field showing partial necrosis of liver cells, infiltration of inflammatory cells and a nodule of regenerating liver cells (bottom right). $\times 80$.

The optic nerves showed no abnormality. The cerebellar cortex showed degenerative changes with Purkinje cell loss and thinning of the granular layer. The dentate nucleus showed changes similar to those found in the thalamus (Fig. 4) and the fibres of the superior cerebellar peduncle showed marked degeneration. In the red nucleus some spongy change was apparent. The inferior olivary nuclei showed pseudohypertrophy (Fig. 5), while the pyramidal tracts stained poorly with myelin stains and showed some ballooned myelin sheaths. The cervical spinal 0 cord showed no abnormality apart from some degeneration of the pyramidal tracts, and there appeared to be loss of occasional anterior horn cells with associated gliosis.

The liver weighed $412 \mathrm{~g}$ (normal $680 \mathrm{~g}$ at this age). The capsular and cut surfaces of the liver were yellow. Histological examination revealed partial 
necrosis of the liver cells, confined principally to the centrilobular regions, and reticulum stains demonstrated collapse and condensation of the reticulum in these areas without significant proliferation. Masson stain revealed very little collagen in these areas suggesting that necrosis had been recent. A diffuse polymorphonuclear infiltrate, and some haemorrhage was associated with necrotic liver cells. In one or two areas the liver cells formed small nodules in which the cells showed polyploidy and intense basophilia indicative of strenuous regenerative activity (Fig. 6). In a few areas there were collections of lymphocytes and plasma cells mainly related to portal tract areas. It was considered that the appearances were similar to those of acute hepatitis with continuing damage to the liver, and in which the presence of regenerative nodules suggested past episodes of injury from which partial recovery had occurred.

\section{DISCUSSION}

Jellinger and Seitelberger (1970) suggest that spongy glio-neuronal dystrophy is a familial degenerative disorder of the nervous system with distinct clinical and pathological findings. Comparison of the clinical and pathological features of the present case with those of Jellinger and Seitelberger (1970) and others cited by them in the literature shows a close similarity, particularly of the pathological findings. There are many pathological features in common, with the main points of similarity being the spongy change in the thalamus and dentate nucleus, the form of the local reaction, and the marked involvement of the cerebral cortex but the sparing of the white matter. The cerebral cortex is less affected in the present case than it was in the patients of Jellinger and Seitelberger. The findings of cortical laminar necrosis, hippocampal degeneration, and cerebellar cortical damage suggest a hypoxic component in the present case. The cerebral cortical lesions are, however, more diffuse than would be expected in a purely hypoxic lesion and superimposition of hypoxia upon a degenerative process would appear more likely. Although the lesions bear some resemblance to those of subacute necrotizing encephalopathy, they lack the intense capillary proliferation found in that condition. This, together with the distribution of the lesions, would appear to distinguish them.

The association of hepatic and cerebral lesions in the present case and case 1 of Jellinger and Seitelberger (1971), as well as in a number of cases previously reported as Alpers' disease or progressive poliodystrophy by Ford, Livingstone, and Pryles (1951), Blackwood, Buxton, Robertson, and Tucker (1963), Dreifuss and Netsky (1964), Laurence and Cavanagh (1968), and Wefring and Lamvik (1967), is a finding of special interest. It raises the question of an aetiological factor common to both cerebral and hepatic lesions. The hepatic lesions are more significant than expected if attributable only to a prolonged debilitating illness. Hepatocerebral degeneration of non-Wilsonian type (Cameron, Jones, Smith, and Wood, 1968) is most unlikely, as the liver changes do not appear to be of a severity necessary to produce cerebral changes of this order over such a long period of time. Also the neuropathological changes are not consistent with this diagnosis. The possibility of a metabolic error affecting both liver and brain needs to be considered. In our patient clinical evidence of liver disease appeared only in the last week of life but the pathological changes present in the liver at necropsy suggested a longer-standing process. Unfortunately, liver function tests were not done in the earlier phase of this patient's illness and it may well be that disturbed liver function tests could provide an earlier clue to the correct diagnosis in such cases. Another possibility that comes to mind with both hepatic and cerebral pathology, especially in view of the resemblance to hepatitis, is viral infection. Some of the neuropathological changes are not dissimilar to those seen in Jacob-Creutzfeldt disease, raising the possibility of a slow virus infection. However, this seems a rather remote possibility in view of the familial nature of the condition with siblings being affected.

Clinical features that are similar to the cases of Jellinger and Seitelberger (1970) include retarded early motor and intellectual development after a normal birth, multifocal and myoclonic seizures, and later blindness. In the present patient and case 1 of Jellinger and Seitelberger the blindness appeared to be of cerebral origin, although case 3 of Jellinger and Seitelberger had a retinal degeneration and optic atrophy. Although these cases and others in the literature reviewed by Jellinger and Seitelberger have a fairly distinct clinical course, it seems that a correct diagnosis cannot be made with certainty on clinical grounds alone. 
Although extensive metabolic investigation in this case has failed to reveal evidence of an inborn error of metabolism, we would favour this as the most likely aetiology. Although there was intolerance to certain foods, it was not possible to identify any specific food component that produced anorexia and gastrointestinal disturbance. Similarly, we were unable to incriminate any particular metabolite in the production of acidosis in association with the episodes of status epilepticus. The familial nature of the illness, with siblings being affected in Jellinger and Seitelberger's (1970) report, together with the likelihood of siblings being similarly affected in the present family, raises the possibility of autosomal recessive inheritance.

We wish to thank Dr. T. G. Maddison who referred this child, Dr. Peter Campbell who carried out the post mortem examination and reported on the pathological changes in the liver, Dr. David Danks for helpful discussion, and Dr. Joseph Dancis who kindly carried out amino acid analysis of urine.

\section{REFERENCES}

Alpers, B. J. (1931). Diffuse progressive degeneration of the gray matter of the cerebrum. Archives of Neurology and Psychiatry, 25, 469-505.
Blackwood, W., Buxton, P. H., Cumings, J. N., Robertson, D. J., and Tucker, S. M. (1963). Diffuse cerebral degeneration in infancy (Alpers' disease). Archives of Disease in Childhood, 38, 193-204.

Cameron, A. H., Jones, E. L., Smith, W. T., and Wood, B. S. B. (1968). Combined hepatic and cerebral degeneration in infancy. Journal of Pathology and Bacteriology, 96, 227-230.

Christensen, E., and Krabbe, K. H. (1949). Poliodystrophia cerebri progressiva (infantilis). Report of a case. Archives of Neurology and Psychiatry (Chic.), 61, 28-43.

Dreifuss, F. E., and Netsky, M. G. (1964). Progressive poliodystrophy. The degenerations of cerebral gray matter. American Journal of Disease in Childhood, 107, 649-656.

Ford, F. R., Livingston, S., and Pryles, C. V. (1951). Familial degeneration of the cerebral gray matter in childhood, with convulsions, myoclonus, spasticity, cerebellar ataxia, choreoathetosis, dementia, and death in status epilepticus. Differentiation of infantile and juvenile forms. Journal of Pediatrics, 39, 33-43.

Jellinger, K., and Seitelberger, F. (1970). Spongy glioneuronal dystrophy in infancy and childhood. Acta Neuropathologica, 16, 125-140.

Klein, H., and Dichgans, J. (1969). Familiäre juvenile glioneurale Dystrophie. Archiv für Psychiatrie und Nervenkrankheiten, 212, 400-422. (Cited by Jellinger and Seitelberger, 1970.)

Laurence, K. M., and Cavanagh, J. B. (1968). Progressixe degeneration of the cerebral cortex in infancy. Brain, 9P 261-280.

Wefring, K. W., and Lamvik, J. O. (1967). Familial pro $\mathbb{D}$ gressive poliodystrophy with cirrhosis of the liver. Acta Paediatrica (Uppsala), 56, 295-300. 